\title{
EDITORIAL
}

\section{Oxidative Stress: An Overview}

\author{
Ahmad Md. Parwez \\ Chief Executive Editor, MED PHOENIX
}

Oxidative stress is defined as a state in which oxidation exceeds the antioxidant systems in the body secondary to a loss of the balance between them. ${ }^{1}$ Free radical is any species capable of independent existence (hence the term 'free') that contains one or more unpaired electrons.

Free radicals are important to any biochemical process and represent a crucial part of aerobic life and our metabolism. They are continuously produced by body's use of oxygen such as in respiration and some cell-mediated immune functions. They are also generated through environmental pollutants, cigarette smoke, automobile exhaust, radiation, air-pollution, pesticides. ${ }^{2}$

The term reactive oxygen species (ROS), often used in the biomedical free radical literature is a collective term that includes not only oxygen-centered radicals and some non-radical derivatives of oxygen.

Oxygen is a very good example of "friend and enemy" as the same molecule is important for existence and is also noxious outside a certain level. Oxygen is critical for aerobic life processes. However about $5 \%$ or more of the inhaled oxygen is converted to reactive oxygen species (ROS). ${ }^{3}$ Though oxygen can behave like a radical (diradical) owing to the presence of 2 unpaired electrons of parallel spin, it does not exhibit extreme reactivity due to quantum mechanical reactions. Its electronic structure results in formation of water by reduction with four electrons i.e

$\mathrm{O}_{2}+4 \mathrm{H}^{+}+4 \mathrm{e}^{-} \rightarrow 2 \mathrm{H}_{2} \mathrm{O}$

The toxicity of oxygen to humans has been of interest in relation to diving underwater, swimming and in the use of hyperbaric oxygen in the treatment of cancer, infections, multiple sclerosis and lung diseases. ${ }^{4}$ High pressure oxygen frequently causes acute central nervous system (CNS) toxicity in animals producing convulsions.

ROS is a collective term, which includes not only the oxygen radicals $(\mathrm{O} 2 \bullet-$, and $\mathrm{OH} \bullet)$ but also some non-radical derivatives of oxygen. These include $\mathrm{H}_{2} \mathrm{O}_{2}, \mathrm{HOCl}$, ozone, singlet oxygen and peroxynitrile.

In spite of endogenous defense mechanisms against ROS whenever there is imbalance between cellular antioxidant system and ROS, oxidative damage to the cells occurs leading to several pathological conditions. 


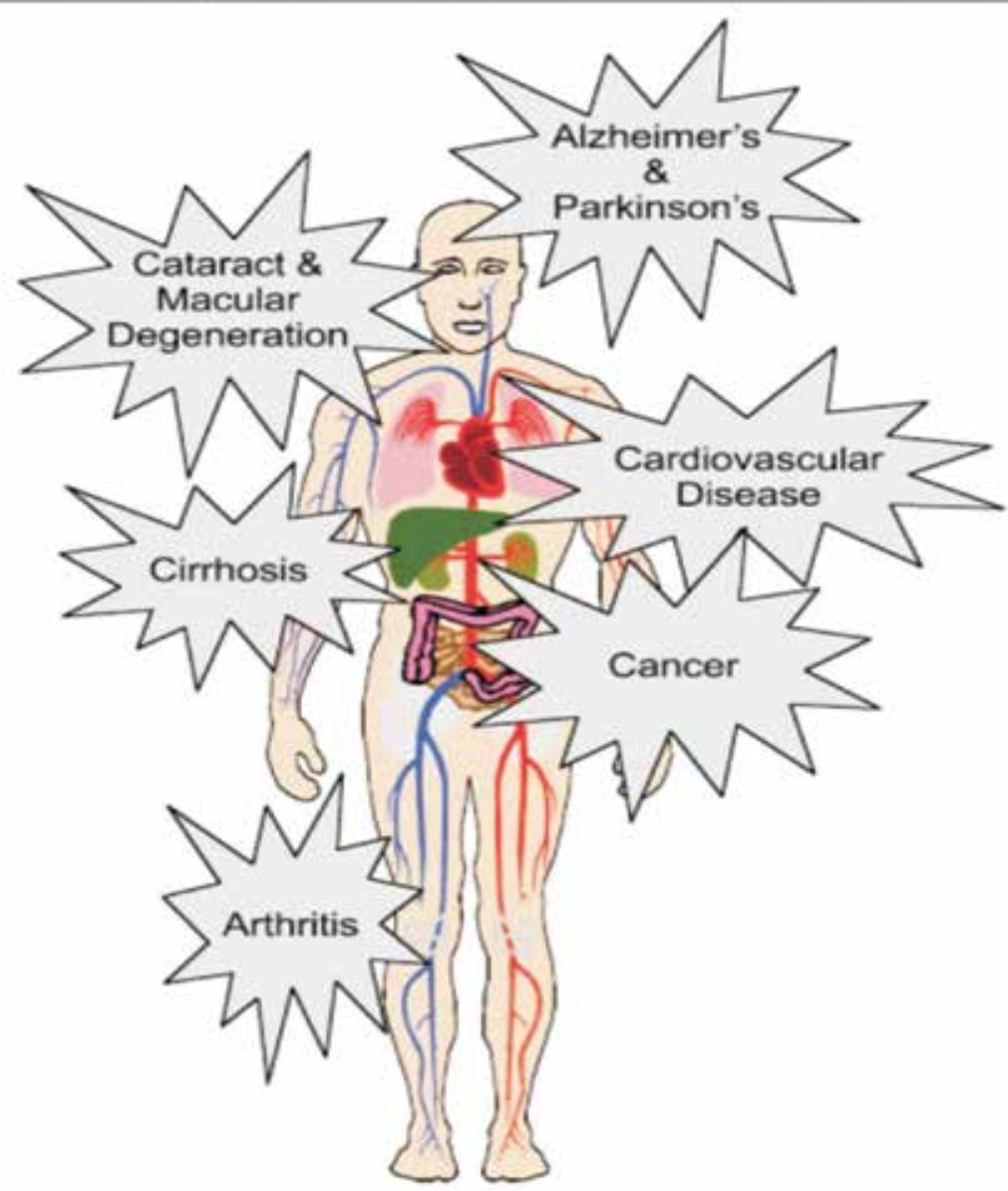

Figure 1 : ROS in diseases (Source: Comparative Biochemistry and Physiology 2003; 136: 113-26)

Over about 100 disorders like rheumatoid arthritis (RA), hemorrhagic shock, CVS disorders, cystic fibrosis, metabolic disorders, neurodegenerative diseases, gastrointestinal ulcerogenesis and AIDS have been reported as ROS mediators. Some specific examples of ROS mediated diseases include Alzheimers disease, Parkinson's disease, Atherosclerosis, Cancer, Down's syndrome and ischemic reperfusion injury in different tissues including heart, liver, brain, kidney and GIT. The role played by ROS in stress induced gastric ulcer and inflammatory bowel diseases have been well established, as well as their involvement in the process of ageing. ${ }^{5}$

\section{REFERENCES}

1. Toshikazu Y, Yuji N. What Is Oxidative Stress. Japan Med Assoc J. 2000; 124(11): 1549-53.

2. Graham JB, Dudley R, Auilar NM, Gans C. Implications of the late Paleozoic oxygen pulse for physiology and evolution. Nature. 1995; 375: 117-20.

3. Broda E. The beginning of photosynthesis. Origins Life. 1975; 6: 247-51.

4. Goel HC, Kumar PI, Rana SV. Free radical scavenging and metal chelation by Tinospora cordifolia a possible role in radioprotection. Ind J Exp Biol. 2002;40: 727-34.

5. Sohal RS, Weindruch R. Oxidative stress, caloric restriction and aging. Science. 1996; 128: 356. 